\title{
Direct Monte Carlo Simulations of a Binary Gas Free-Jet Flow Over a Flat Plate
}

\author{
Eric Cecil \\ Department of Mechanical and Aerospace Engineering, University of Virginia, Charlottesville, VA 22903, USA
}

\begin{abstract}
Monte Carlo simulations of low density, hypersonic free-jet wind tunnel tests [1] are presented for comparison with test measurements, made by a laser-induced iodine fluorescence method, of Mach 12 flow over a flat plate at zero incidence. The radially expanding free-jet core is modeled as a 3-dimensional (spherical) expansion. Upstream velocity, temperature, and density profiles were established directly from the Ashkenas-Sherman correlations for jet-centerline Mach number and off-axis density. The flow simulations extended to the free-jet wake and ambient test pressure and include the characteristic 'Mach stem' jet termination feature. The test gas was composed of a binary mixture of nitrogen with a trace quantity of iodine, and this composition was maintained in an axisymmetric flow simulation to establish the influence of diffusive separation on the accuracy of the test measurements. Velocity profiles of both species on the plate are compared to the measured iodine velocity profiles. Mole fraction profiles of iodine are presented. Rotational and translational temperature profiles of iodine from the simulation are compared with test measurements.
\end{abstract}

\section{INTRODUCTION}

A Monte Carlo simulation was developed to simulate a low density, hypersonic flow encountered in laboratory measurements of a slip-regime flow over a flat plate which had been obtained by laser-induced fluorescence (LIF) of iodine seeded in the wind tunnel gas [2]. The simulations are intended to better understand the laboratory flow and specifically the ability of iodine seed molecules to "track" the nitrogen carrier gas in the wind tunnel in terms of measuring velocity, temperature, and density. A second goal was to develop a compact model to simulate a free jet wind tunnel with a minimal computational domain, while including the effect of background pressure in the vacuum chamber producing the free jet.

Figure 1 is a narrowband iodine PLIF image of flow over a flat plate model mounted in the U.Va. free-jet wind tunnel. Light areas in the image indicate spatial fluorescence intensity patterns. The fluorescence serves here to visualize the oblique shock attached to the plate leading edge, a Mach stem structure, and the barrel shock of the jet.

\section{DESCRIPTION OF THE FLOW FIELD MODEL}

Boundary conditions for the axisymmetric Monte Carlo simulation are presented in figure 2. Both upstream and downstream surfaces were represented by 'in-flow' boundaries. The bounding streamlines were modeled as solid walls producing specular reflection of incident molecules. The flat plate surface is modeled as an isothermal solid wall producing diffuse reflections of incident molecules, with complete thermal accomodation to the surface temperature. The isothermal surface temperature $T_{s}$ was taken equal to the stagnation temperature $T_{o}$.

The upstream condition on the jet axis was determined by the Ashkenas-Sherman Mach number correlation and isentropic flow relations from the jet stagnation conditions $\left(T_{o}=302.7 \mathrm{~K}, p_{o}=1.824 \times 10^{5} \mathrm{~N} / \mathrm{m}^{2}\right)$. Density at a given radius in the core of a free jet is known to be reduced away from the jet axis. Ashkenas and Sherman found this behavior to be approximately self-similar and developed the following empirical correlation for the angular variation of density from method-of-characteristics solutions [3].

$$
\rho(r, \theta)=\rho(r, 0) \cos ^{2}\left(\frac{\pi \theta}{2 \phi}\right)
$$

where $r=\sqrt{x^{2}+y^{2}+z^{2}}, \theta=\arctan \left(\sqrt{x^{2}+z^{2}} / y\right)$, and the fitting parameter $\phi=1.662$ for a gas of specific heat ratio $\gamma=1.4$.

For isentropic flow, the temperature and radial velocity variations are 


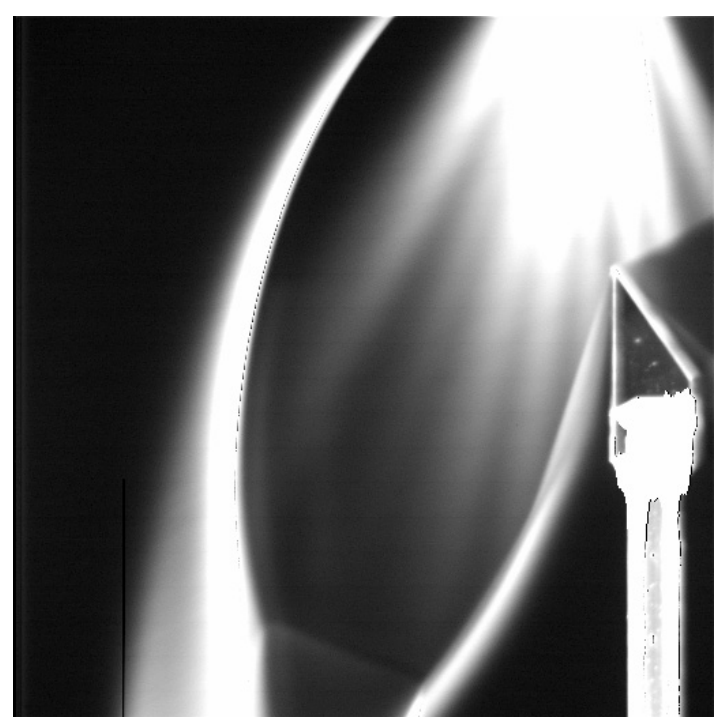

FIGURE 1. $I_{2}$ PLIF image of hypersonic flow over a flat plate model [2].

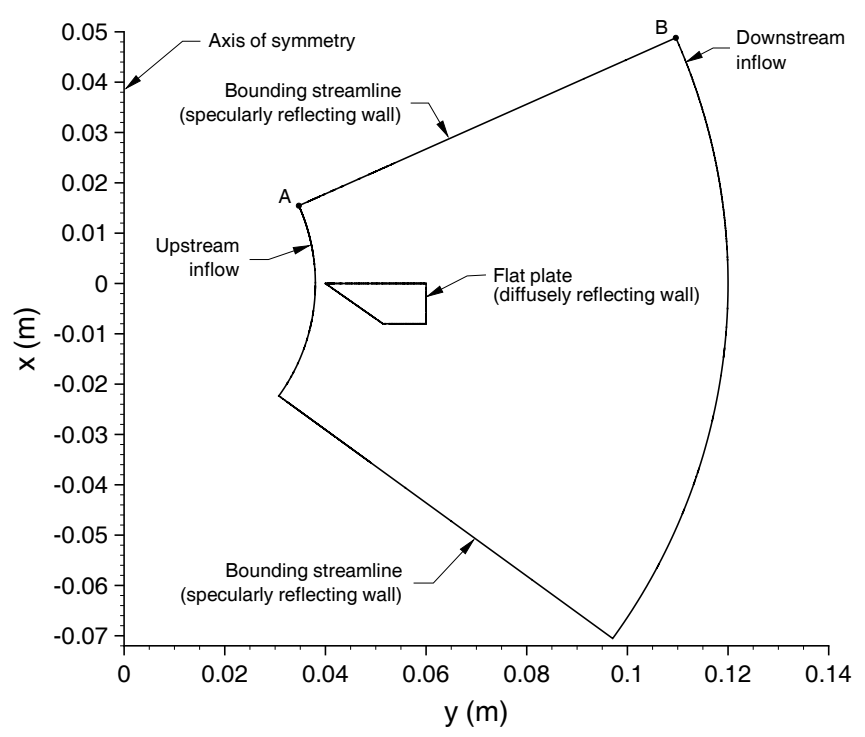

FIGURE 2. Boundary conditions for the axisymmetric Monte Carlo simulation.

$$
T(r, \theta)=T(r, 0)\left(\frac{\rho(r, \theta)}{\rho(r, 0)}\right)^{\gamma-1}
$$

and

$$
V_{r}(r, \theta)=\sqrt{V_{r}(r, 0)^{2}+\frac{2 \gamma R_{\mathrm{u}}}{(\gamma-1) \mathfrak{M}}(T(r, 0)-T(r, \theta))} .
$$

To minimize the computational domain, an axisymmetric DSMC model was developed. The $x$-axis was chosen as the symmetry axis rather than the jet centerline on the $y$-axis so that a flat plate surface is possible. This simplification permits modeling the undisturbed upstream portion of the jet as a spherical source-like flow, but implies that the flat plate has curved leading and trailing edges. Furthermore, using the $x$-axis as an axis of symmetry means all gradients out of the $x-y$ plane are zero. (A $2 \mathrm{~d}$ simulation, on the other hand, would produce a cylindrical jet expansion with the wrong expansion rate, so that the Mach stem location would not correlate with the observation in figure 1.)

The downstream boundary location was selected based on LIF images such as figure 1 which indicated that an outer radius of $120 \mathrm{~mm}$ should be sufficient to enclose the Mach stem which meets the oblique shock developed by the flat plate in the jet core. A back pressure of about $280 \mathrm{mTorr}\left(37.3 \mathrm{~N} / \mathrm{m}^{2}\right)$ was measured in the LIF experiment. Uniform pressure at the downstream condition is sufficient to ensure development of a Mach stem to recompress all flow undisturbed by the plate. For an in-flow condition in the DSMC, pressure must be applied by simultaneously choosing appropriate values of number density, temperature, and velocity at the boundary.

Consider a spherically-symmetric flow with a spherical shock wave-in this case the spherical shock corresponds to a Mach stem or Mach disk in the free jet. By applying conservation of mass for spherical flow $\left(n r^{2} V_{r}=\right.$ constant), the ideal gas law, and the inviscid, adiabatic, perfect gas energy equation, the following expression for the downstream velocity on a radial streamline through the Mach stem was developed:

$$
\left(\frac{\gamma-1}{2}\right) \frac{\mathfrak{M}}{\gamma R_{u} T_{o}} V_{r, B}^{2}+\frac{p_{B}}{n_{A} k T_{o}}\left(\frac{r_{B}}{r_{A}}\right)^{2} \frac{V_{r, B}}{V_{r, A}}-1=0 .
$$

The downstream velocity $V_{r, B}$ may be found approximately by this equation, when $p_{B} \approx p_{o, B}$, which then allows determination of the remaining properties at point $\mathrm{B}$. These values were calculated using the points $\mathrm{A}$ and $\mathrm{B}$ shown in the figure, and applied uniformly to the downstream boundary.

The specularly-reflecting walls, representing radial streamlines passing undeflected through Mach stems, bound the flow at $24^{\circ}$ off axis on the flat plate side and $-36^{\circ}$ on the opposite side, and were chosen somewhat qualitatively. 


\section{GAS MOLECULE COLLISION MODEL}

In the present simulations the representation of iodine molecules has been increased from a laboratory value of roughly one in 10,000 to one in 1,000 in order increase the number of samples of the iodine specie and thereby reduce scatter. This resulted in approximately $10^{4}$ samples of iodine molecule properties in each cell, which was adequate to bring statistical scatter to a tolerable level. The increase in proportion of iodine was kept small enough so that the molar mass of the gas mixture is altered by less than $1 \%$, and therefore the effect on bulk flow behavior should be negligible.

The variable hard sphere (VHS) molecular diameter model was used for determining collision cross-sections. Data for nitrogen was taken from the gas property tables in appendix A of Bird's text. For iodine, a VHS molecular diameter and viscosity law exponent were determined from vapor viscosity data $\left(d=6.5 \times 10^{-10} \mathrm{~m}, \omega=0.981\right.$ at $\left.T_{\text {ref }}=473 \mathrm{~K}\right)$.

Rotational energy interactions in collisions are simulated using the Larsen-Borgnakke phenomenological model. The number of collisions for rotational relaxation in $\mathrm{N}_{2}-\mathrm{N}_{2}$ collisions was taken to be 5, which is considered typical. The characteristic rotational temperature of nitrogen, $2.9 \mathrm{~K}$, is assumed to be sufficiently low relative to the $11 \mathrm{~K}$ upstream condition (and the developing expansion) so that the rotational modes are fully excited.

The number of collisions for rotational relaxation of an $\mathrm{I}_{2}$ molecule in $\mathrm{I}_{2}-\mathrm{N}_{2}$ collisions was taken to be 10 . This value amounts to a guess, due to lack of information. (Perhaps the value should be higher, closer to 50, based on the molecular mass difference between $\mathrm{I}_{2}$ and $\mathrm{N}_{2}$.) The DAC97 program does not allow setting this value independently from a value for $I_{2}-I_{2}$ collisions, so both values must be 10 . Because $I_{2}$ is a trace gas, the $I_{2}-I_{2}$ collisions will be relatively very rare, however, and there is no special concern about them at the present concentration.

Vibrational modes were assumed to be essentially frozen out and not significant.

\section{SIMULATION DETAILS AND ADAPTATIVE REFINEMENT}

The present simulation was computed using the DAC97 computer program on a Linux cluster using 48 processors running for about 4-5 days.

The steady state DSMC 'solution' is developed from molecule property samples taken after the decay of initially unsteady behavior in a time-marching flow simulation. The initial condition of the flow field was a perfect vacuum, with simulated gas molecules entering at the upstream and downstream in-flow boundaries.

In the time-marching simulation, the time step must be much less than the local mean time between collisions for a molecule, $\Delta t_{c}$. A 'global' time step of $10^{-8} \mathrm{~s}$ was employed and assumed to be sufficiently small relative to $\Delta t_{c}$ throughout. 100,000 steps were allowed for initialization before sampling, and completed with 300,000 steps.

A uniform 'level I' Cartesian grid was used with $1196 \times 894$ cells $10^{-4} \mathrm{~m}$ square. An adaptative refinement of the simulation parameters was performed and the simulation was repeated. The adaptation produced a refined 'level II' grid which increased the number of cells in regions where the uniform 'level I' grid size exceeded the local mean-freepath. This resulted in some grid refinement in the vicinity of the shock waves. In performing the adaptation, the DAC97 program also changes the cell-based weighting factors for the ratio of the number of real-to-simulated molecules in an attempt to produce a uniform number of simulated molecules per cell.

Cumulative samples of the number of simulated molecules and molecular properties $\left(u, v, w, u^{2}, v^{2}, w^{2}, u v, v w, u w\right.$, etc.) were taken every other time step at each cell in double-precision.

\section{SIMULATION RESULTS AND DISCUSSION}

Contour lines of $\mathrm{N}_{2}-\mathrm{I}_{2}$ mixture density (normalized by the nominal value at the leading edge) are plotted in figure 3 . An oblique shock forms at the flat plate leading edge, develops increasing angle curving into the jet stream, and is reflected at the upper flow field boundary by a Mach reflection pattern. A long Mach stem compresses the undisturbed portion of the jet, and a shear flow develops along the slipstream issuing from the triple point, where the shock formed by the plate, the Mach stem, and the reflected shock all intersect. Number density is reduced near the surface of the plate and develops into a relatively uniform value, to within about $20 \%$, in the separated wake of the plate. The oblique shock curvature and Mach stem appear qualitatively consistent with the LIF flow visualization of these features in figure 1.

Streamlines and contour lines of the mean flow speed are plotted in figure 4. Boundary layer separation takes place on the flat plate surface at approximately $3 / 4$ of the plate length. This result is in disagreement with the velocity measurements presented in [2], which showed attached flow for the full plate length. 

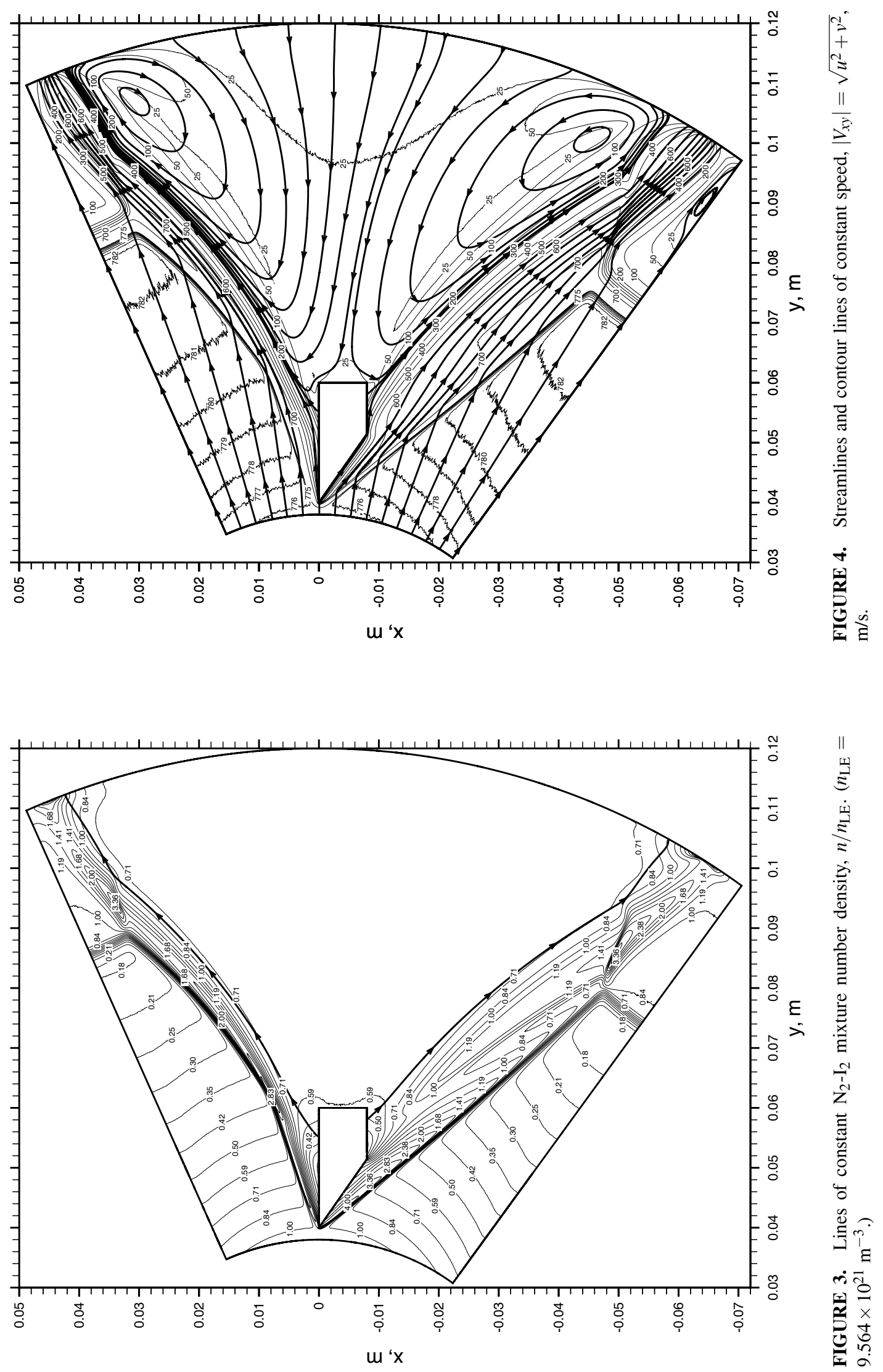


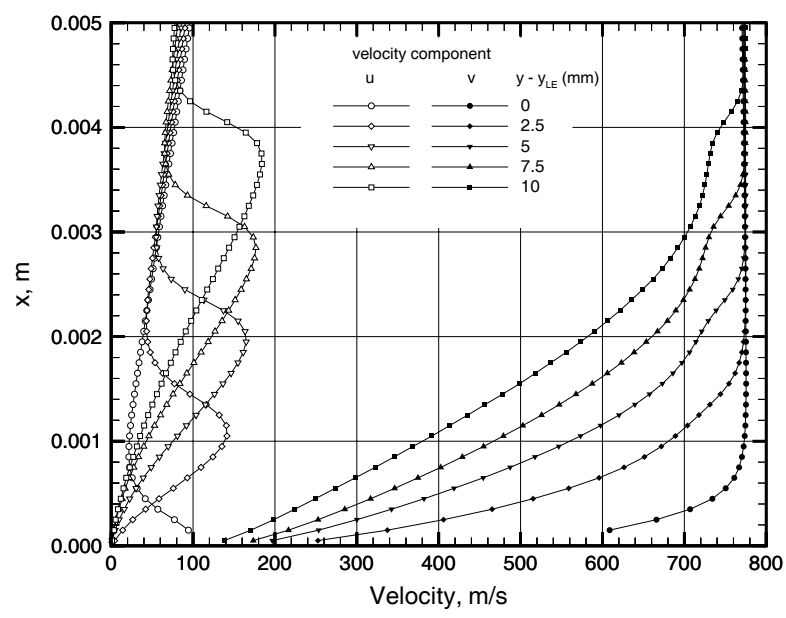

FIGURE 5. Profiles of $\mathrm{N}_{2}$ velocity components $u$ and $v$ at several stations along the plate. $y-y_{\mathrm{LE}}$ is distance from the plate leading edge.

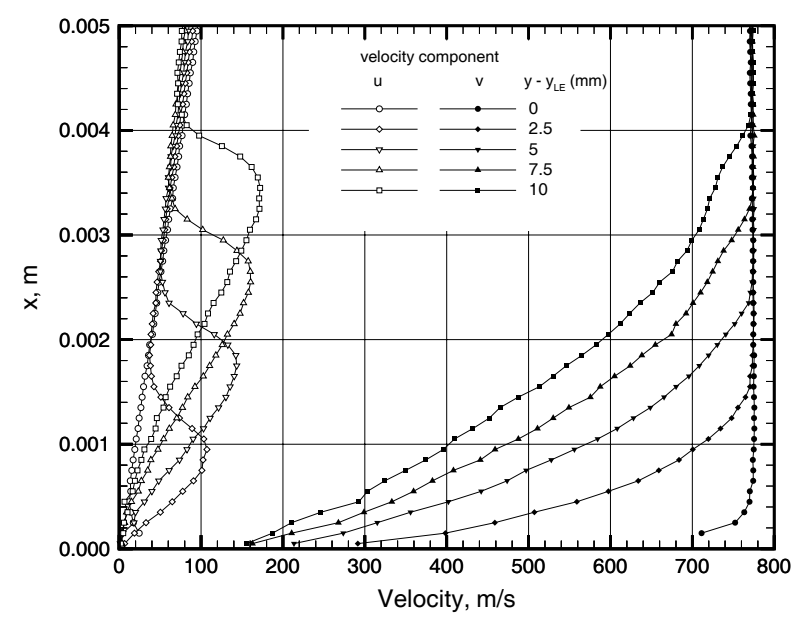

FIGURE 6. Profiles of $\mathrm{I}_{2}$ velocity components $u$ and $v$ at several stations along the plate. $y-y_{\mathrm{LE}}$ is distance from the plate leading edge.

The flow separation in the simulation appears to be caused by the relatively simple nature of the uniform downstream boundary condition. Shear layers emerging from the Mach reflection patterns impinge with a velocity of roughly $600 \mathrm{~m} / \mathrm{sec}$ on the downstream boundary which has been specified to be much slower. This impingement evidently causes some of the shear layer gas to be diverted parallel to the downstream boundary and thereby entrain gas into the counter-rotating vortices in the wake. The most obvious sign of this problem is at the bottom of figure 4 , where a small vortex has formed on the specularly reflecting wall, just ahead of the downstream boundary.

Profiles of $x$ and $y$ component velocity of the $\mathrm{N}_{2}$ and $\mathrm{I}_{2}$ species on the flat plate are plotted in figures 5 and 6 . Symbols in the plots indicate individual DSMC cell data. The $\mathrm{I}_{2}$ velocity profiles suggest that it is capable of keeping close pace with the $\mathrm{N}_{2}$, except within the shock fronts. The $\mathrm{I}_{2}$ shock front profiles are displaced slightly closer to the plate relative to the $\mathrm{N}_{2}$ profiles. This behavior of the $\mathrm{I}_{2}$ in the DSMC gives better agreement with the $\mathrm{I}_{2}$ PLIF velocity measurements in [2].

Profiles of $x, y$, and $z$ components of the $\mathrm{N}_{2}$ and $\mathrm{I}_{2}$ translational temperature at a location midway along the flat plate are plotted in figures 7 and 8 .

For the axisymmetric flow of figure 2, the $z$-axis is a principal direction by symmetry. In the $x-y$ plane, translational temperature about an arbitrarily rotated axis may be obtained using the ideal gas law and a 2-d transformation of the pressure tensor components. Consider an axis $x^{\prime}$ which is rotated from $x$ by an angle $\psi$ about the $z$-axis:

$$
T_{x^{\prime}}=\frac{T_{x}+T_{y}}{2}+\frac{T_{x}-T_{y}}{2} \cos (2 \psi)+\frac{p_{x y}}{n k} \sin (2 \psi) .
$$

Figure 9 is a plot of $T_{x^{\prime}}$ of $\mathrm{I}_{2}$ at a location $10 \mathrm{~mm}$ along the plate for $\psi=-45^{\circ}$. This profile compares well qualitatively with recent $\mathrm{I}_{2}$ LIF measurements at that laser incidence angle in a related flow [4].

Several normalized mole fraction profiles of $\mathrm{I}_{2}$ are presented in figure 10 and indicate the presence of local regions of significant depletion and enrichment in $\mathrm{I}_{2}$. The possibility of such variations must be taken into account in interpreting density measurements taken from $\mathrm{I}_{2}$ LIF data.

\section{ACKNOWLEDGMENTS}

Professor J. C. McDaniel served as faculty advisor. This research was supported by: the Aerothermodynamics Branch at NASA Langley Research Center, Peter Gnoffo, technical monitor, and Christopher E. Glass; Ball Aerospace; NASA Marshall Space Flight Center; and the Virginia Space Grant Consortium. The simulations were performed using the DAC97 computer software provided by Gerald J. LeBeau of NASA Johnson Space Center. The simulations were carried out on the U.Va. ITC Linux clusters. 


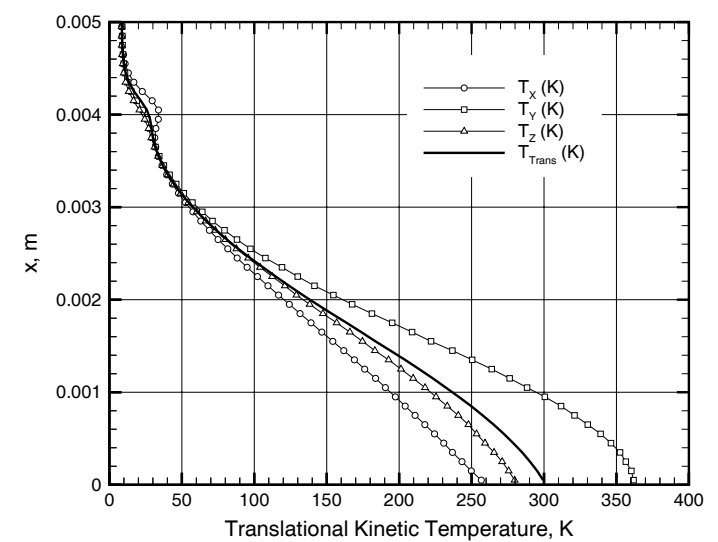

FIGURE 7. Profiles of the $x, y, z$ translational modes and the mean translational temperature of $\mathrm{N}_{2}$ at location $10 \mathrm{~mm}$ from the plate leading edge.

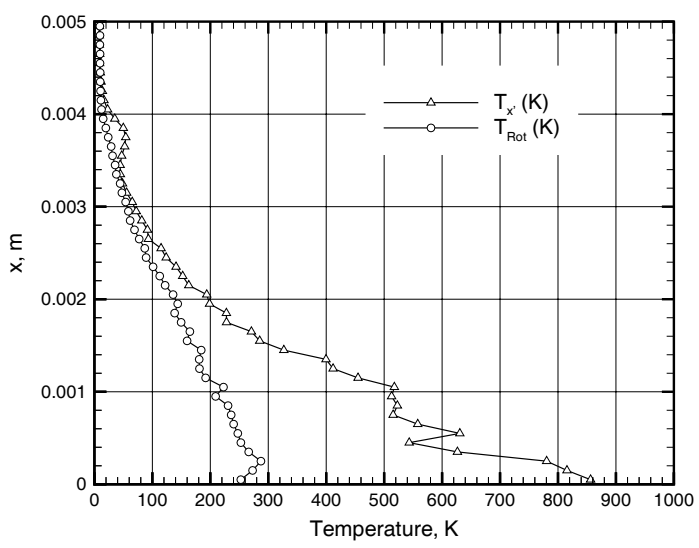

FIGURE 9. Profiles of the $x^{\prime}$ translational and rotational temperature of $\mathrm{I}_{2}$, located $10 \mathrm{~mm}$ from the plate leading edge.

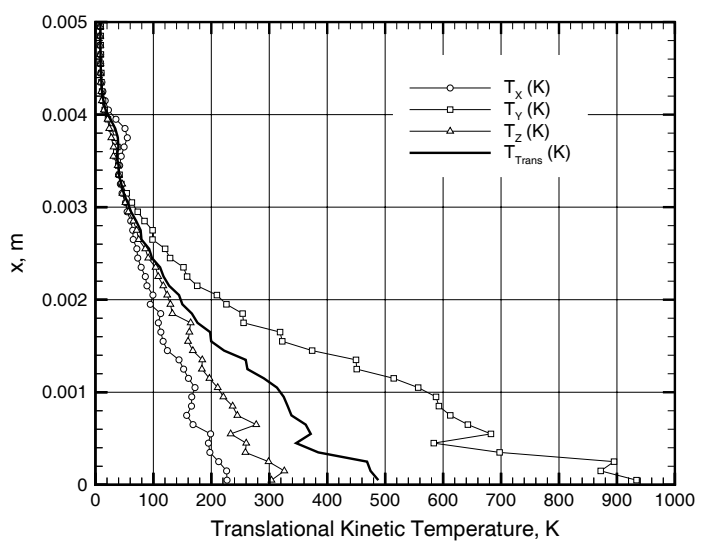

FIGURE 8. Profiles of the $x, y, z$ translational modes and the mean translational temperature of $\mathrm{I}_{2}$ at location $10 \mathrm{~mm}$ from the plate leading edge. Note the temperature scale.

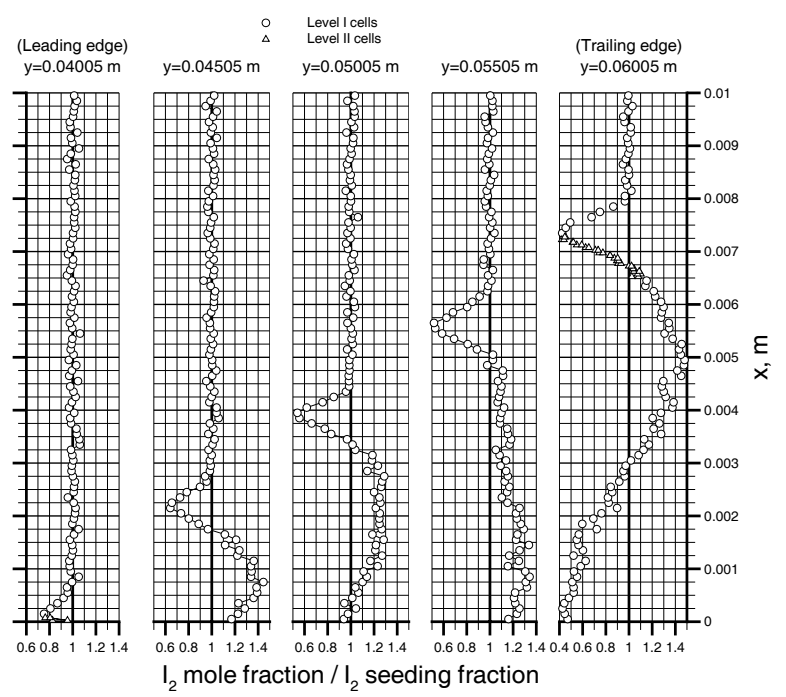

FIGURE 10. Profiles of the $I_{2}$ mole fraction normalized by the $I_{2}$ seeding fraction at several locations along the plate.

\section{REFERENCES}

1. D. E. Cecil, Ph.D. dissertation, University of Virginia, expected 2011.

2. D. E. Cecil and J. C. McDaniel, "Planar Laser-Induced Iodine Fluorescence Measurements in Rarefied Hypersonic Flow" in AIP Conf. Proc. 762. Ed. M. Capitelli, AIP, 2005, pp. 1325-1330.

3. H. Ashkenas, and F. S. Sherman, "The Structure and Utilization of Supersonic Free Jets in Low Density Wind Tunnels" in Advances in Applied Mechanics, Supplement 3, Vol. 2, Academic Press, New York, 1966.

4. D. E. Cecil and J. C. McDaniel, "Flow Measurements of Translational-Rotational Nonequilibrium Using Laser-Induced Iodine Fluorescence.” 27th International Symposium on Rarefied Gas Dynamics, Pacific Grove, California, 2010. 